\title{
Dipping in Cygnus X-2 in a multi-wavelength campaign due to absorption of extended ADC emission
}

\author{
M. Bałucińska-Church ${ }^{1}$, N. S. Schulz ${ }^{2}$, J. Wilms ${ }^{3}$, A. Gibiec ${ }^{4}$, M. Hanke ${ }^{3}$, R. E. Spencer ${ }^{5}$, \\ A. Rushton ${ }^{5,6,7}$, and M. J. Church ${ }^{1}$ \\ ${ }^{1}$ School of Physics \& Astronomy, University of Birmingham, Birmingham, B15 2TT, UK \\ e-mail: mbc@star.sr.bham.ac.uk \\ 2 Kavli Institute for Astrophysics and Space Research, Massachusetts Institute of Technology, Cambridge MA 02139, USA \\ 3 Dr. Karl Remeis-Sternwarte, Astronomisches Institut der Universität Erlangen-Nürnberg, Sternwartestrasse 7, 96049 Bamberg, \\ Germany \\ 4 Astronomical Observatory, Jagiellonian University, ul. Orla 171, 30-244 Cracow, Poland \\ 5 Jodrell Bank Centre for Astrophysics, School of Physics \& Astronomy, University of Manchester, Manchester M13 9PL, UK \\ ${ }^{6}$ Onsala Space Observatory, 43992 Onsala, Sweden \\ 7 European Southern Observatory, Karl-Schwarzschild-Strasse 2, 85748 Garching, Germany
}

Received 14 October 2010 / Accepted 22 March 2011

\section{ABSTRACT}

\begin{abstract}
We report results of one-day simultaneous multiwavelength observations of Cygnus X-2 using XMM, Chandra, the European VLBI Network and the $X M M$ Optical Monitor. During the observations, the source did not exhibit Z-track movement, but remained in the vicinity of the soft apex. It was in a radio quiescent/quiet state of $<150 \mu \mathrm{Jy}$. Strong dip events were seen as $25 \%$ reductions in X-ray intensity. The use of broadband CCD spectra in combination with narrow-band grating spectra has now demonstrated for the first time that these dipping events in Cygnus X-2 are caused by absorption in cool material in quite a unique way. In the band 0.2-10 keV, dipping appears to be due to progressive covering of the Comptonized emission of an extended accretion disk corona, the covering factor rising to $40 \%$ in deep dipping with an associated column density of $3 \times 10^{23}$ atom $\mathrm{cm}^{-2}$. Remarkably, the blackbody emission of the neutron star is not affected by these dips, in strong contrast with observations of typical low mass X-ray binary dipping sources. The Chandra and XMM gratings directly measure the optical depths in absorption edges such as $\mathrm{Ne} \mathrm{K}, \mathrm{Fe} \mathrm{L}$, and $\mathrm{O} \mathrm{K}$ and a comparison of the optical depths in the edges of non-dip and dip data reveals no increase of optical depth during dipping even though the continuum emission sharply decreases. Based on these findings, at orbital phase 0.35 , we propose that dipping in this observation is caused by absorption in the outer disk by structures located opposite to the impact bulge of the accretion stream. With an inclination angle $>60^{\circ}$ these structures can still cover large parts of the extended ADC, without absorbing emission from the central neutral star.
\end{abstract}

Key words. accretion, accretion disks - acceleration of particles - stars: neutron - X-rays: binaries -

$\mathrm{X}$-rays: individuals: Cyg X-2 - radio continuum: general

\section{Introduction}

Z-sources form the brightest group of low mass X-ray binaries (LMXB) containing accreting neutron stars with superEddington luminosities between $2-6 \times 10^{38} \mathrm{erg} \mathrm{s}^{-1}$. Strong physical changes take place within the sources as is shown by the three spectral tracks traced in X-ray hardness-intensity diagrams known as the horizontal branch (HB), the normal branch (NB) and flaring branch (FB) (Schulz et al. 1989; Hasinger \& van der Klis 1989). The six original Galactic Z-sources are divided into the Cygnus X-2 like sub-group (Cygnus X-2, GX 340+0 and GX 5-1), in which complete Z-shapes are normally seen, and the Sco X-1 like sub-group (Scorpius X-1, GX 17+2 and GX 349+9) in which the HB is small or missing but in which flaring is stronger (Hasinger \& van der Klis 1989; Kuulkers \& van der Klis 1995). It is important to understand the formation of relativistic jets in these sources. In Sco X-1, radio observations with the VLA revealed the outward motion of radio condensations at $v \sim 0.45 c$ (Fomalont et al. 2001). The radio detection is on a restricted part of the Z-track near the hard apex between HB and NB. When X-ray bright, the highly variable LMXB Cir X-1 exhibited Z-source behaviour (Shirey et al. 1998), a powerful
X-ray accretion disk wind (Brandt \& Schulz 2000), and relativistic jet emission (Fender et al. 2004; Heinz et al. 2007). Thus $\mathrm{X}$-ray observations of Z-sources uniquely allow the study of conditions connecting the inner disk and the neutron star to mechanisms of jet formation.

The nature of the Z-track phenomenon is not understood. However, recent studies have produced models that can explain spectral changes along the Z-track, notably the extended accretion disk corona (ADC) emission model of Church \& Bałucińska-Church (2004). This is based on long-term studies of the dipping LMXBs in which absorption in the bulge in the outer disk causes dips in X-ray intensity at the orbital cycle (White \& Swank 1982). Spectral evolution during dipping can be explained in terms of point-like blackbody emission from the neutron star and the dominant Comptonized emission of an extended ADC (Church \& Bałucińska-Church 1997). The extended nature of the ADC is shown by measurement of dip ingress times giving sizes of 20000-700000 km depending on luminosity (Church \& Bałucińska-Church 2004). Strong support for an extended ADC comes from Chandra high-resolution grating results (Schulz et al. 2009) for highly excited H-like lines of $\mathrm{Ne}, \mathrm{Mg}, \mathrm{Si}, \mathrm{S}$ and $\mathrm{Fe}$ in Cygnus X-2, with Doppler widths 
corresponding to radii between 18000 and $110000 \mathrm{~km}$ in good agreement with dip ingress timing.

The extended ADC model produces a straightforward explanation of the Z-track in the Cyg X-2 like sources (BałucińskaChurch et al. 2010). The large increase in the luminosity of the ADC emission $\left(L_{\mathrm{ADC}}\right)$ suggests that the mass accretion rate $(\dot{M})$ increases between soft and hard apex, opposite to previous suggestions (Hasinger et al. 1990) in which $\dot{M}$ decreases. The neutron star flux increases from a fraction of Eddington at the soft apex to super-Eddington at the hard apex and HB. The resulting high radiation pressure at the inner disk diverts accretion flow vertically launching the jets observed on this part of the Z-track. The FB consists of unstable nuclear burning of the accretion flow as suggested by the good agreement of the mass accretion rate per unit area with the critical $\dot{m}_{\mathrm{ST}}$ when burning becomes unstable (Bildsten 1998).

RXTE observations of a huge outburst in XTE J1701-462 (Lin et al. 2009) show the source evolving from a superEddington Cyg X-2 like source to Sco X-1 like, and finally into an Atoll source. The interpretation was that $\dot{M}$ changes caused the luminosity decrease, but did not drive Z-track motion. However, the observations did not produce much interpretation for the nature of the Z-track, and in the spectral model used the Comptonized emission was only $10 \%$ of the total luminosity contrary to general acceptance that this is much higher in LMXB sources.

Cygnus X-2 is the archetypal Z-source. For a distance of 8-11 kpc (Cowley et al. 1979; Smale 1998) it is thought that there is an evolved companion of $0.4-0.7 M_{\odot}$. Recent highresolution optical spectroscopy of Casares et al. (2010) provides a refined orbital solution with a period of $9.84450 \pm 0.00019 \mathrm{~d}$. Assuming an inclination of $62.5 \pm 0.4^{\circ}$ from ellipsoidal fits to UBV lightcurves (Orosz \& Kuulkers 1999), Casares et al. found a neutron star mass of $1.71 \pm 0.21 M_{\odot}$, supporting a previous result (Casares et al. 1998), while Elebert et al. (2009) derived $1.5 \pm 0.3 M_{\odot}$.

Many observations of Cyg X-2 indicate an extended ADC. Vrtilek et al. (1988) suggested that high and low flux states reflect changes in the geometrical and optical thickness of the accretion disk and ADC. H-like Fe xxvi line emission was found by Smale et al. $(1993,1994)$ using $B B X R T$; lines from various K-shell ions were found (Kuulkers et al. 1997; di Salvo et al. 2002) using ASCA and BeppoSAX. Recent Chandra observations revealed a wealth of detail: $\mathrm{K}$-shell emission with lines from H-like and He-like ions of Mg to Fe (Schulz et al. 2009). The fully-resolved broad lines gave Doppler velocities indicating radii of more than $10^{9} \mathrm{~cm}$ (as detailed above) and plasma densities of $\sim 10^{15} \mathrm{~cm}^{-3}$ consistent with an origin in a hot, dense, extended ADC.

Cygnus X-2 often exhibits short-term decreases (dips) in $\mathrm{X}$-ray intensity generally described as a feature of the FB unrelated to absorption, first recognized by Bonnet-Bidaud \& van der Klis (1982). Hasinger et al. (1990) showed the reduced intensity intervals in two forms. In hardness-intensity, the FB is usually short or absent, while dipping comprises reductions in intensity from positions near the soft apex. But in hard colour versus soft colour, the same dip data form a third track looking exactly like an extensive FB. Thus, there was confusion whether dipping was absorption or flaring, and showing the use of colour-colour diagrams may mislead because these do not distinguish between decreasing and increasing intensity. Clarifying this has not previously been carried out. The assumed association of dipping and flaring led to the idea that inclination was the main difference between Cyg-like and Sco-like sources: it was argued that in Cyg X-2 and GX 340+0, the FB corresponds to X-ray dips and that the Cyg-like group have high inclination allowing absorption in the inner disk inflated by radiation pressure (Kuulkers \& van der Klis 1995); in the Sco-like sources the inclination is small. This assumes that $\dot{M}$ is maximum on the FB causing disk inflation, but Bałucińska-Church et al. (2010) proposed that $\dot{M}$ is minimal on the FB in the Cyg-like sources.

There has since been little discussion of the nature of the dipping/flaring. In the present work, we resolve this, showing that dipping is not associated with the FB. We present results of long simultaneous observations of Cyg X-2 with XMM and Chandra aimed at investigating changes of the continuum and lines around the Z-track. Simultaneous high-resolution radio observations were conducted, aimed at detecting relativistic jets, if present during our campaign, and possibly monitor the ejection of plasma blobs from the source.

\section{Observations and data analysis}

We observed Cygnus X-2 in a multi-wavelength campaign on 2009 May 12-13, starting at 9:30 UT. Both XMM-Newton and Chandra were used in essentially simultaneous one-day observations. In addition, the Optical Monitor onboard XMM provided optical data in the UVW1 band, i.e. in the band 2500-4000.

High-resolution interferometric radio data were obtained using the e-VLBI mode of the European VLBI network (e-EVN) at $5 \mathrm{GHz}$. Observations were approved following a target-ofopportunity (ToO) request and were scheduled on 2009 May $12-13$ to coincide with the multi-wavelength campaign.

\subsection{XMM-Newton EPIC-pn}

The EPIC-pn camera (Turner et al. 2001) was operated in burst mode, with the thin filter because the high flux of the source $\left(>1.3 \times 10^{-8} \mathrm{erg} \mathrm{s}^{-1} \mathrm{~cm}^{-2}\right)$ would result in strong pile-up. In burst mode, it is arranged that the photon collection efficiency (the live time) is reduced to $3 \%$ allowing observations of bright sources. After correcting for the live time, Cygnus X-2 produced a count rate of $\sim 3000$ count $\mathrm{s}^{-1}$, very much less than the pileup limit of 60000 count $\mathrm{s}^{-1}$. In burst mode, spatial information is only available in the $x$-direction, perpendicular to the CCD readout direction. This mode provides high time resolution of $7 \mu$ s and a moderate energy resolution of $E / \Delta E \sim 40$ at $7 \mathrm{keV}$.

Analysis of the EPIC-pn data was carried out using the SAS 9.0.0 software and calibration data from April 1, 2010. A barycentric correction was applied to the event file using BARYCEN and also a correction for the charge transfer inefficiency (CTI) effect seen in EPIC-pn fast mode data using EPFAST; without this correction an unreal feature will remain in the spectrum at $\sim 2 \mathrm{keV}$.

Lightcurves were extracted from the events file by selections made in the one-dimensional image using evselect. During extraction, standard screening was applied to use single and double events and remove known hot pixels. In the pseudo onedimensional image the $y$-axis for RAWY $<180$ represents time and a lightcurve can be derived from this. The part of the $y$-axis used was further restricted to RAWY $<140$ based on the tests made by Kirsch et al. (2006) in observations of the Crab pulsar and the Crab nebula, also using EPIC-pn data in burst mode. Using the Crab nebula as a calibration source, they showed that data between RAWY $=140-180$ should not be used to prevent pulse pile-up affecting not only the slope, but also the normalization of the spectrum. Source data were extracted from a strip 
25 pixels wide centred on the source at pixel 38. Background was obtained in two strips, each 5 pixels wide, one centred on pixel 5 and the other at pixel 62, as far from the source as possible on each side of the source.

To check for the presence of flaring particle background due to protons in the Earth's magnetosphere, a lightcurve was extracted using all RAWX values in the $10-12 \mathrm{keV}$ energy range with single events only (PATTERN $=0$ ). Periods of increased background activity were detected in this way, but at the level of 0.3 count $\mathrm{s}^{-1}$ and so totally negligible in comparison with the source intensity.

Various corrections were made using EPICLCCORR including those for vignetting, bad pixels and the point spread function; this program also corrected for the $3 \%$ live time by scaling the intensity to the value it would have for $100 \%$ live time, and background was then subtracted. A background-subtracted and deadtime-corrected lightcurve was extracted in the total energy band $0.2-10.0 \mathrm{keV}$ and also in several sub-bands $0.5-2.5 \mathrm{keV}$, $2.5-4.5 \mathrm{keV}, 4.5-8.5 \mathrm{keV}$ used for derivation of hardness ratios as described below.

Spectra were extracted with EvSELECT using single and double events. Response functions consisting of the response matrix file (rmf) and ancillary response file (arf) were built using RMFGEN and ARFGEN.

\subsection{XMM MOS}

The MOS detectors could not be used because of the source brightness, and so both detectors MOS1 and MOS2 were blanked across the central CCD so that no useful data on Cygnus X-2 were obtained.

\subsection{XMM RGS}

The standard mode of the Reflecting Grating Spectrometer (RGS; den Herder et al. 2001) reads out eight CCDs of RGS 1 (via two nodes) and eight CCDs of RGS 2 (via a single node), resulting in accumulation times of $4.8 \mathrm{~s}$ and $9.6 \mathrm{~s}$, respectively. Photon pile-up in the dispersed spectrum can be an issue and we therefore aimed with the RGS observation at the spectral range above $20 \AA$, which is not as well covered by the Chandra High Energy Transmission Grating Spectrometer (HETGS). The readout sequence of four CCDs only on each RGS was optimised such that the frame time of the CCD receiving the highest flux on each RGS was reduced to a quarter of the default value. This allowed us to monitor the oxygen edge at low count rates per frame time free of pile-up.

The RGS data were analysed with XMM SAS 9.0.0 software, following standard procedures: RGSPROc was used to extract spectra and create response files, and RGSLCCORR to create exposure-corrected light curves in the full $0.33-0.60 \mathrm{keV}$ (20.7-37.6 ̊) band, as well as in the $0.33-0.52 \mathrm{keV}$ (23.8-37.6 $\AA$ ) band below the $\mathrm{O}$ edge, and in the $0.55-$ $0.60 \mathrm{keV}(20.7-22.5 \AA)$ band above the the edge and associated $\mathrm{O}$ I resonance absorption lines.

\section{4. $X M M O M$}

The Optical Monitor (Mason et al. 2001) was used in "Image Fast" mode and the ultraviolet filter UVW1 was used, this filter giving the best sensitivity in the desired wavelength range of 2500-4000 $\AA$. The B filter was not used because of two bright stars in the field of view. The observation was divided into exposures $4400 \mathrm{~s}$ long, giving coverage of almost all the X-ray observations and providing a lightcurve with $10 \mathrm{~s}$ binning.

Data from the Optical Monitor (OM) were analysed using standard procedures. Firstly, tracking corrections were applied using the tools OMPREP, OMDRIFTHIST and OMTHCONV. Flat-fielding was carried out using OMFASTFLAT, and in the final step lightcurves were produced using OMREGION, EVSELECT for source and background selection, and OMLCBUILD.

\subsection{Chandra HETGS}

The Chandra observations with the High Energy Transmission Grating spectrometer spanned about $67 \mathrm{ks}$ beginning at 13:00 UT on May 12 and had very good overlap with the XMM exposures. Because of the extreme brightness of the source in combination with the very narrow point spread function, strong photon pile-up is expected for normal CCD frametime operations. Thus we chose the fastest available detector readout time (3.82 $\mathrm{ms})$ provided in continuous clocking (CC) mode as done in a previous Chandra observation of the source (Schulz et al. 2009).

All data were analysed using CIAO4.2 and the most recent CALDB products. The first order count rates were quite similar during the two observations, averaging about 220 count s $^{-1}$ leading to an average value for the counts/frametime/node of $\sim 0.025$, and for the counts/frametime/pixel, a value well below 0.01 . We are therefore not worried about pile-up in the HETG spectra. Bright sources observed in CC mode have different charge transfer inefficiency properties and have to be treated somewhat differently with respect to the normal timed event mode (see Schulz et al. 2009).

\subsection{Radio observations}

Cygnus X-2 was observed using the e-EVN from 2009 May 12 23:52:22 UT until 2009 May 13 12:54:03 UT at $5 \mathrm{GHz}$. The telescopes participating were Jodrell Bank MkII, Knockin, Cambridge, Effelsberg, Torun, Yebes, Medicina, Onsala 25-m and Sheshan. Data were transferred in real time from each antenna to the correlator using high-speed dedicated e-VLBI network links ${ }^{1}$; connection rates of up-to $1024 \mathrm{Mbps}$ were sustained per antenna, giving a maximum bandwidth of $256 \mathrm{MHz}$. The field of view was centred on $\alpha=21^{\mathrm{h}} 44^{\mathrm{m}} 41^{\mathrm{s}} .2, \delta=38^{\circ} 19^{\prime} 18^{\prime \prime} .0$ and phased reference to the calibrator source J 2134+4050. Data were then reduced using the standard AIPS VLBI algorithms and pipelined using EVN scripts written in PARSELTONGUE (i.e. a python wrapper for classical AIPs).

\section{Radio results}

The target was not detected. The noise level of the image was $\sim 30 \mu \mathrm{Jy}$, hence the source had a five sigma upper limit of $\sim 150 \mu \mathrm{Jy}$ per beam; the source was therefore in a radio-quiet state. Hjellming et al. (1989) presented VLA results as part of the 1988 multi-wavelength campaign on Cygnus X-2. Detailed correlations of radio flux at $1.49,4.9$ and $8.4 \mathrm{GHz}$ with X-ray intensity and $\mathrm{X}$-ray hardness showed that the source had a radio flux of 2-5 mJy when the source was on the horizontal or upper normal branch, but fell to a low level $<1 \mathrm{mJy}$ on the lower normal and flaring branches, which was designated as a radio-quiet

${ }^{1}$ e-VLBI developments in Europe are supported by the EC DG-INFSO funded Communication Network Developments project "EXPReS", Contract No. 02662. 

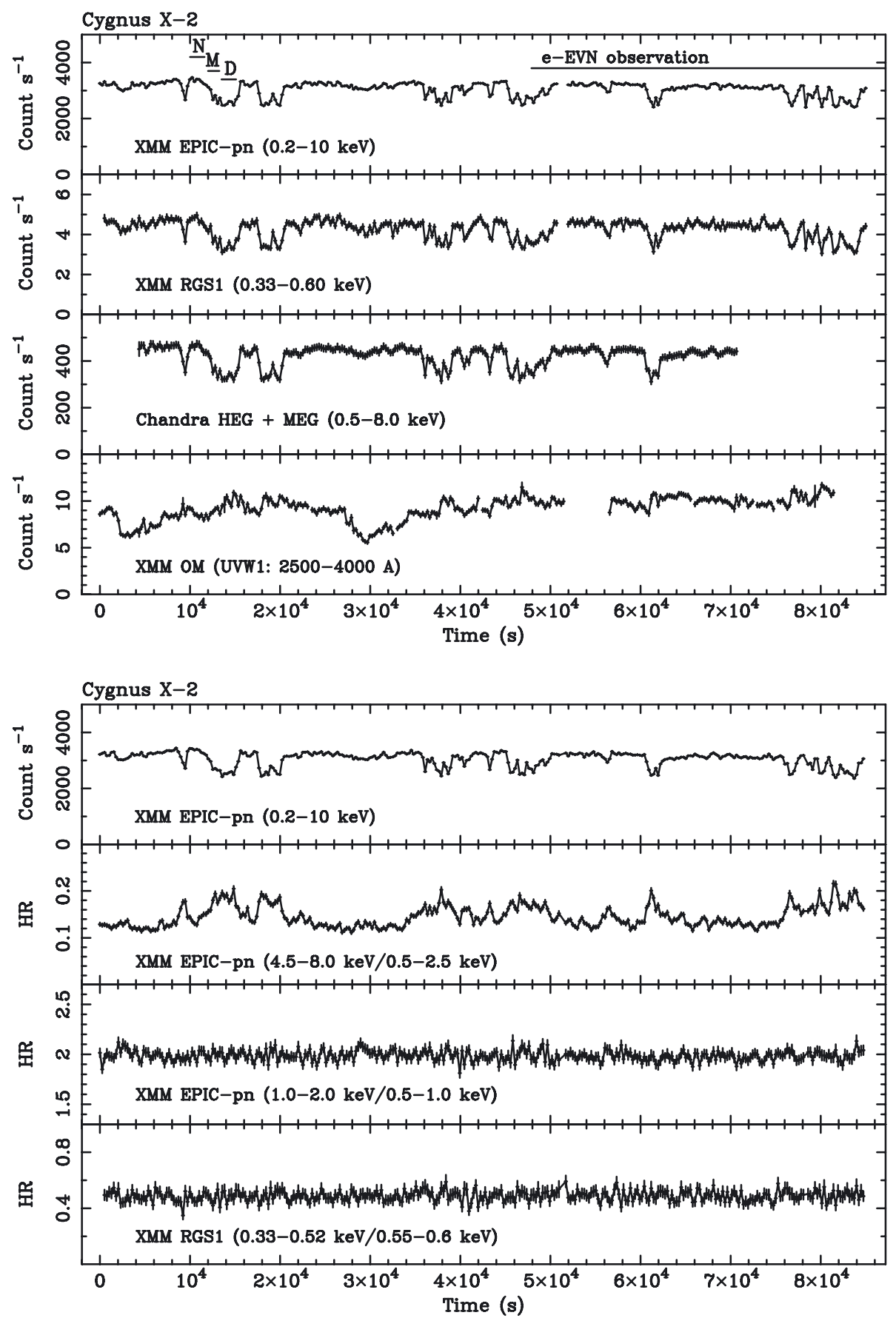

Fig. 1. Upper panel: background-subtracted and deadtime-corrected EPIC-pn light curve of Cygnus X-2 in the band 0.2-10.0 keV; spectra of non-dip, medium and deep dipping were selected from the sections labelled "N", "M" and " $\mathrm{D}$ ". Upper middle panel: the corresponding 0.33-0.60 keV RGS1 lightcurve; lower middle panel: light curve of the Chandra HETGS HEG + MEG instruments in first order; lower panel: the $X M M$ Optical Monitor lightcurve in the UVW1 band; all four lightcurves have $256 \mathrm{~s}$ binning.
Fig. 2. Hardness ratios defined in several energy bands: upper middle panel: using wide EPIC-pn bands; lower middle band: using EPIC-pn bands below $2 \mathrm{keV}$; lower panel: using the RGS1 band. The upper panel gives the 0.2-10 keV EPIC-pn lightcurve for comparison. state. The present X-ray results indicate the source to be in a stationary position on the Z-track at or close to the soft apex, and so the lack of radio detection is consistent with this. The radio results have been published in Astronomer's Telegram 2052 by Rushton et al. (2009).

\section{X-ray results}

In Fig. 1 (upper panel) we show the XMM-Newton EPIC-pn lightcurve with $256 \mathrm{~s}$ binning together with the corresponding RGS1 lightcurve (upper middle panel), the Chandra HETG lightcurve (lower middle panel) and the OM lightcurve (lower panel). It can be seen that the $X M M$ observation spanned $86 \mathrm{ks}$, while the Chandra observation was somewhat shorter at $67 \mathrm{ks}$. All X-ray lightcurves clearly display reductions in intensity of up to $25 \%$, having the appearance of absorption dips as seen in the dipping class of LMXBs. The dipping is structured but seems to have a consistent lower level, or maximum depth of dipping. In addition to this variability, there is a continuous small decrease in the non-dip intensity across the observation from $\sim 3400$ to $\sim 3300$ count $\mathrm{s}^{-1}$.

\subsection{Hardness ratios}

The source changes were investigated by the use of several X-ray hardness ratios from the EPIC-pn and RGS instruments as shown in Fig. 2. The upper panel shows the EPIC-pn $0.2-10 \mathrm{keV}$ lightcurve for comparison with the upper middle panel giving a hardness ratio defined as the ratio of counts per second in the band $4.5-8.0 \mathrm{keV}$ to that in the band $0.5-2.5 \mathrm{keV}$, these bands being chosen to allow comparison with Chandra data, which do not extend to $10 \mathrm{keV}$. The lower middle panel shows a hardness 
XMM PN

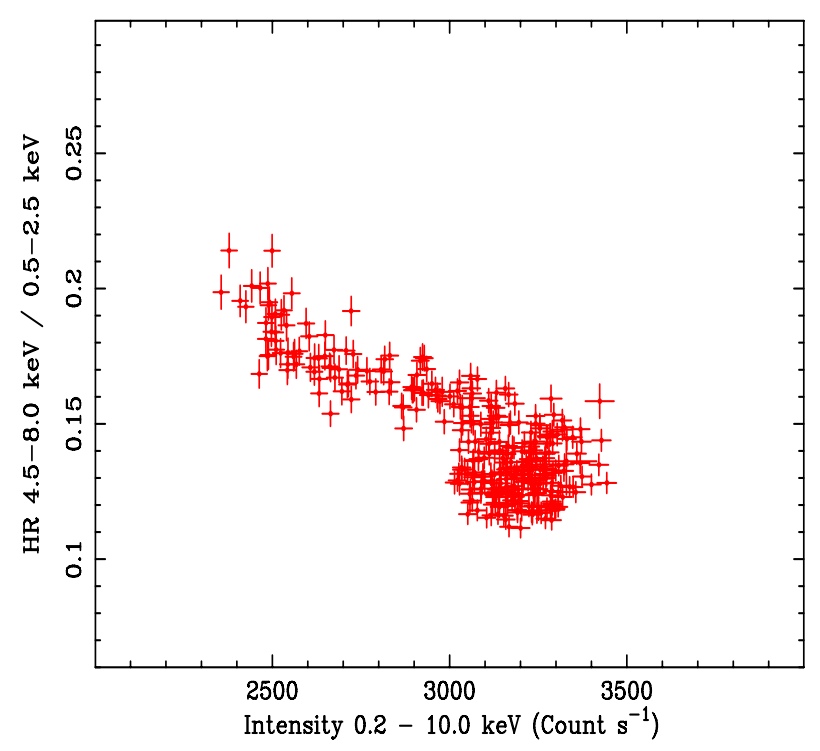

Fig. 3. Hardness-intensity diagram for the $X M M P N$ data.

ratio from EPIC-pn defined using the bands $1.0-2.0 \mathrm{keV}$ and $0.5-1.0 \mathrm{keV}$, in the low energy part of the spectrum and the lower panel shows a similar ratio from RGS1 using the bands $0.33-0.52 \mathrm{keV}$ and $0.55-0.60 \mathrm{keV}$. The hardness ratio based on the wide PN band shows a factor of two hardening of the spectrum during the dips which is the behaviour demonstrated in many dipping LMXB sources, reflecting the tendency for absorption to remove the lower energies; however, this is not always the case as the spectrum consists of two emission components: the neutron star and the ADC, which may have different degrees of absorption (Church et al. 1997). The hardness ratios defined in bands below $2 \mathrm{keV}$ remarkably show no change in hardness, which is a result of the nature of the absorbing process, which will be discussed in Sects. 4.3 and 5.2.

The source variations were investigated further by displaying the $X M M$ EPIC-pn data on a hardness-intensity diagram as shown in Fig. 3. The same hardness ratio was used as in Fig. 2 (upper middle panel). Sections of data in the lightcurve were identified in the hardness-intensity diagram by making a number of selections of short sections of data.

This revealed that the non-dip data form an approximately circular soft region at the right hand side of the diagram. Data of decreased intensity form the trail extending to lower intensities and harder parts in Fig. 3. It can be seen that the source did not move on its Z-track, but remained in a fixed position during the observations. Spectral fitting described below shows that the decreases of intensity are indeed absorption events, i.e. X-ray dips with column density increases, as opposed to the possibility of this trail being part of the horizontal branch of the Z-track.

\subsection{Spectral fitting of Epic-pn spectra}

Spectra were extracted by selection on times and intensities from the EPIC-pn lightcurve corresponding to non-dip, medium dip and deep dip data (labelled N, M and D in Fig. 1). Using the section of strong dipping near the start of the observations (at 10-15 ks), a non-dip spectrum was selected having intensity $>3400$ count $\mathrm{s}^{-1}$, i.e. that part of the EPIC-pn lightcurve at times between 10-12 ks. An intermediate dip spectrum was taken with intensities between 2970-3120 count $\mathrm{s}^{-1}$ corresponding to times of $\sim 12-13 \mathrm{ks}$, and a deep dip spectrum at intensities $<2460$ count $^{-1}$, at times of $\sim 13-16 \mathrm{ks}$. The non-dip spectrum was obtained as close to the dip data as possible to avoid any drift in source intensity. Spectra were re-grouped to effectively oversample the spectrum by a factor of three at each energy. This required primitive channels to be binned in groups of five below $1.0 \mathrm{keV}$, in groups of eight between $3-4 \mathrm{keV}$, and in tens between $7-8 \mathrm{keV}$ etc. Spectra were fitted in the range $0.6-12.0 \mathrm{keV}$.

These spectra were fitted using the extended ADC emission model so that the non-dip spectrum was fitted by a model of the form $\mathrm{ABS} *(\mathrm{BB}+\mathrm{CUT})$, where $\mathrm{ABS}$ is the Galactic absorption, BB the neutron star blackbody emission and cuT the Comptonized emission of an extended ADC (Church \& Bałucińska-Church 2004). In fitting, the Xspec model PHABs was used as a more accurate form than the generally used wABs model. A background spectrum was obtained from the strips at each side of the CCD (Sect. 2.1) using the whole observation, and allowance made for the difference in area using BACKSCAL. In the Z-track sources, the Comptonization high energy cut-off is relatively low at about $5 \mathrm{keV}$, which does not allow determination of the power law photon index given the narrow available energy range. Accordingly, the index was fixed at the physically reasonable value of 1.7 (Shapiro et al. 1976) as we have previously carried out as standard (Church et al. 2006).

In preliminary testing, it became evident that dipping consisted of absorption of the Comptonized emission only, and to demonstrate this, the non-dip and deep dip spectra were fitted simultaneously by a model in which each emission term had a scaling factor: $\mathrm{K} 1 * \mathrm{BB}+\mathrm{K} 2 * \mathrm{cUT}$. For the non-dip spectrum, $\mathrm{k} 1$ and $\mathrm{k} 2$ were frozen at unity. Fitting showed that during dipping, k1 remained closely equal to unity: $\mathrm{k} 1=1.01 \pm 0.05$ at $90 \%$ confidence but $\mathrm{k} 2$ decreased to $\sim 0.60$, indicating that the Comptonized emission was removed while the blackbody was not affected. This contrasts markedly with spectral evolution in the dipping LMXB in which the blackbody is absorbed with a much higher column density that the Comptonized emission, indicating that the neutron star emission is subject to absorption along a path of denser material through central parts of the bulge in the outer disk (Church et al. 1997). Absorption could be seen to take place at energies below $5 \mathrm{keV}$, demonstrating that photoelectric absorption is taking place. The neutron star blackbody spectrum, however, peaks at $\sim 3 \mathrm{keV}$ so that its flux at $1 \mathrm{keV}$ is relatively small comprising only $10 \%$ of the total flux. At $0.5 \mathrm{keV}$ this falls to $1 \%$, and it is clear that absorption of the blackbody cannot explain the dipping and that the predominant absorption process is absorption of the Comptonized emission (see Fig. 4).

From this point onwards, fitting was carried out using a model of the form $\mathrm{ABS} *(\mathrm{BB}+\mathrm{PCF} * \mathrm{CUT})$ where $\mathrm{ABS}$ is Galactic interstellar absorption and $\mathrm{PCF}$ is a progressive covering factor as appropriate to removal of extended emission by an extended absorber passing across the line-of-sight. In analysis of the dipping LMXB, spectral evolution in dipping could be well-described by a model in which the neutron star blackbody was absorbed and the ADC emission was progressively covered by absorber (e.g. Church et al. 1997), i.e. a model ABS*(ABS*BB + PCF*CUT) representing the effects of the absorbing bulge on the outer disk passing across the neutron star, and also progressively overlapping the extended ADC. The difference in the present case is the lack of strong absorption of the neutron star blackbody emission.

The non-dip, intermediate dip and deep dip spectra were fitted with this model. It was possible to fit the non-dip spectrum first and then apply the emission parameters found to the dip spectra, or alternatively, to fit the three spectra simultaneously. 
XMM EPIC-pn

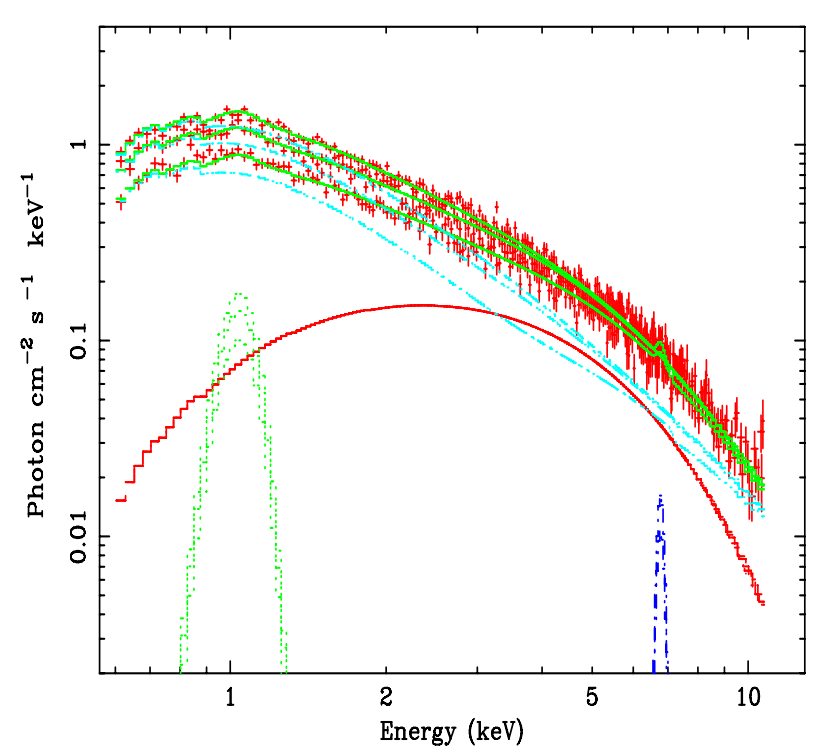

Fig. 4. Best fit to the non-dip, intermediate dip and deep dip spectra showing in each case the data, total model, blackbody and Comptonized emission components and the emission lines at 1 and $6.6 \mathrm{keV}$. It can be seen that in the best-fit model, the blackbody curve peaking at $3 \mathrm{keV}$ does not change during dipping as it is not subject to absorption.

Both techniques provided good fits, and we present here results of simultaneous fitting. In this case, the progressive covering factor was frozen at zero for the non-dip spectrum, but was free for each of the dip spectra. Emission parameters were chained between all three spectra, forcing them to be equal. With the above continuum model, a broad excess could be seen in the residuals at $1 \mathrm{keV}$ and a weak feature at $\sim 6.6 \mathrm{keV}$. Testing showed that the $1 \mathrm{keV}$ feature was reduced in flux in dipping and could be modelled in dipping by including the Gaussian line inside the partial covering factor, this suggesting that the emission originated in the ADC. The Fe feature was weaker and its behaviour in dipping could not be constrained.

A good simultaneous fit was obtained having $\chi^{2} /$ d.o.f. $=$ $633 / 588=1.08$ and when each spectrum was individually examined, the fit was good in each case (see Table 1).

The blackbody emission was found to have a temperature $k T_{\mathrm{BB}}$ of $1.29 \pm 0.07 \mathrm{keV}$, and a blackbody radius $R_{\mathrm{BB}}$ of $(9.6 \pm 1.1) \times(d / 8 \mathrm{kpc}) \mathrm{km}$ for a source distance of $8 \mathrm{kpc}$. The Comptonized emission was well described by a cut-off power law with power law index 1.7 and cut-off energy $8.9 \pm 1.9 \mathrm{keV}$; all uncertainties are quoted at $90 \%$ confidence. This cut-off energy is higher than the values of $4-5 \mathrm{keV}$ that we have previously obtained for the Z-track sources, presumably because we can only fit up to $12 \mathrm{keV}$ in the present case. The Galactic column density $N_{\mathrm{H}}^{\mathrm{Gal}}$ was found to be $(3.6 \pm 0.1) \times 10^{21}$ atom $\mathrm{cm}^{-2}$ assuming the cross sections of Verner et al. (1996) and the abundances of Wilms et al. (2000), substantially higher than the value of $(2.3 \pm 0.5) \times 10^{21}$ atom $\mathrm{cm}^{-2}$ of Juett et al. (2004). The total 1-10 keV luminosity of the source was found to be $L_{\text {Tot }}=9.83 \times 10^{37} \mathrm{erg} \mathrm{s}^{-1}$ for a distance of $8 \mathrm{kpc}$ (Cowley et al. 1979).

To better obtain the line parameters, a spectrum was constructed containing all EPIC-pn non-dip data (excluding the features at $\sim 3$ and $\sim 30 \mathrm{ks}$ (Sect. 4.5 ), and using this the $1 \mathrm{keV}$ feature was approximately described by a Gaussian line at $1.03 \pm 0.03 \mathrm{keV}$ and width $\sigma=0.09 \pm 0.03 \mathrm{keV}$ with equivalent
Table 1. Spectral evolution in dipping.

\begin{tabular}{lccc}
\hline \hline Spectrum & $f$ & $N_{\mathrm{H}}$ & $\chi^{2} /$ d.o.f. \\
\hline Non-dip & 0.0 & 0.0 & $223 / 194$ \\
Intermediate dip & $0.182 \pm 0.019$ & $13_{-6}^{+13}$ & $187 / 192$ \\
Deep dip & $0.421 \pm 0.015$ & $45_{-12}^{+20}$ & $223 / 192$ \\
\hline
\end{tabular}

Notes. Column densities are given in units of $10^{22}$ atom $\mathrm{cm}^{-2}$.

width of $28 \mathrm{eV}$. Close examination of the Fe feature revealed structure which could be fitted by two Gaussian lines; however, it was necessary to freeze each $\sigma$ at $30 \mathrm{eV}$ to represent the width seen in the residuals. The line energies were found to be $6.61 \pm 0.04 \mathrm{keV}$ and $6.88 \pm 0.05 \mathrm{keV}$ with EW of 18 and $17 \mathrm{eV}$ indicative of states Fe XXV and Fe XXVI in agreement with the values obtained by Schulz et al. (2009) of $6.662 \pm 0.018$ and $6.919 \pm 0.035 \mathrm{keV}$. Schulz et al. resolved the Fe XXV Helike triplet and the Fe XXVI H-like resonance line with moderate line widths that correspond to $3450 \mathrm{~km} \mathrm{~s}^{-1}$ and $1120 \mathrm{~km} \mathrm{~s}^{-1}$, respectively. This indicated formation at a radius much greater than $10^{9} \mathrm{~cm}$ from the neutron star. Done et al. (2002) claimed that the broad Fe feature in Ginga observations of Cyg X-2 was well described as associated with reflection in the accretion disk of a central Comptonizing region around the neutron star. Similarly, Shaposhnikov et al. (2009) fitted Suzaku data with a disk reflection model. The HETG spectra of Cyg X-2 so far only show contributions from ionized Fe lines of moderate broadening. Contributions from neutral Fe fluorescence can be ruled out. Fe fluorescence is only seen in a minority of LMXB; the HMXB Cyg X-3 in Chandra clearly exhibits Fe K- $\alpha$ fluorescence and the hot photoionization lines Fe XXV and Fe XXVI (Torrejón et al. 2010). The Chandra spectra of Cyg X-2 indicate emissions from the outer part of the system rather than reflection from the inner disk.

The intermediate dip and deep dip spectra were well described by the above emission parameters together with the partial covering factors and column densities shown in Table 1. The covering factor term PCF may be written as $f * \mathrm{ABS}+(1-f)$, i.e. a fraction $f$ of the Comptonized emission is subject to additional absorption with column density $N_{\mathrm{H}}$ (as shown in Table 1) while the remainder of the emission $(1-f)$ is not overlapped by absorber. It can be seen that the covering factor rises to $40 \%$ in deep dipping with a high column density of $45 \times 10^{22}$ atom $\mathrm{cm}^{-2}$ which strongly removes ADC emission below $5 \mathrm{keV}$. This can be seen in Fig. 4, which shows the unfolded spectra and the best-fit models obtained by simultaneous fitting.

Finally a check was made of whether the neutron star blackbody emission could be subject to a small amount of photoelectric absorption in addition to the strong absorption of the Comptonized emission. This was done by adding an additional column density to the blackbody: $\mathrm{ABS} * \mathrm{BB}$, frozen at zero for the non-dip spectrum, but free in the two dip spectra. Free fitting showed that this extra column density remained at zero in both intermediate and deep dip spectra (in deep dipping $N_{\mathrm{H}}=$ $0_{-0.0}^{+1.7} \times 10^{22}$ atom $\mathrm{cm}^{-2}$ ). There was no change in the quality of fit with $\chi^{2} /$ d.o.f. $=633 / 586$. This may be contrasted with fitting the dipping group of LMXB in which the Comptonized emission is progressively covered, often reaching $100 \%$ covering, but in which the neutron star blackbody is also absorbed, and it is typically found that $N_{\mathrm{H}}^{\mathrm{BB}}$ is very high having a lower limit of the order of $600 \times 10^{22}$ atom $\mathrm{cm}^{-2}$ (e.g. Church et al. 1997). It is 
clear that in the present case, absorption of this component is negligible.

\subsection{XMM RGS results}

The RGS exhibited a similar lightcurve to those of the EPICpn and the Chandra gratings, clearly showing the X-ray dips. Spectral fitting of the available RGS band (0.33-0.60 keV) to determine spectral evolution in dipping cannot be sensibly carried out because of the narrowness of the band. However, it is possible to investigate the effect of dipping on the oxygen edge. In Fig. 2 (lower panel) we show a hardness ratio from RGS data defined as the ratio of counts in the bands $0.33-0.52 \mathrm{keV} / 0.55-0.60 \mathrm{keV}$, i.e. bands on either side of the oxygen edge. Thus, these energy bands indicate not only any hardness change in the RGS band, but also whether there is any increased absorption in the oxygen edge at the time of dipping. No change at all is evident, which is consistent with the EPIC spectral fitting results. This fitting showed that the X-ray emission in dipping consists of the covered part of the emission, which is subject to a high column density of absorber, plus the uncovered emission, which is not absorbed at all. In the RGS band the flux of the covered emission is negligible and the emission detected consists entirely of the uncovered emission, which has essentially the non-dip spectrum scaled down by a factor $(1-f)$. This result is confirmed by the hardness ratio in Fig. 2 obtained from EPIC data using counts in the bands $1.0-2.0 \mathrm{keV} / 0.5-1.0 \mathrm{keV}$, which also shows no change during dipping, as expected. In fact, the EPIC-pn spectrum in non-dip divided by the dip spectrum is constant below $2.0 \mathrm{keV}$ demonstrating that the total removal of the covered emission produces an energy-independent reduction in the spectral flux below $2 \mathrm{keV}$, and thus the low energy hardness ratios in Fig. 2 are accurately constant in dips.

Spectral fitting was carried out to determine absorption in the oxygen edge. Because of the reduced count statistics compared with the EPIC-pn, it was necessary to use longer sections of data and a dip spectrum was accumulated consisting of all degrees of dipping (25 ks exposure) and a spectrum consisting of all non-dip data $(58 \mathrm{ks})$. A parameterization of the local continuum was used consisting of two power laws. The first order spectra of RGS1 and RGS2 were rebinned to $S / N=15$ in the band 20.8-37.3 $\AA$. The absorption edge of neutral oxygen and the fine structure due to the related series of resonance absorption lines, in particular the strong $1 \mathrm{~s}-2 \mathrm{p}$ transition of neutral oxygen at $\sim 23.5 \AA$, were modelled by a local absorption model (TBNEW) assuming the cross sections of Verner et al. (1996) and the abundances of Wilms et al. (2000). The non-dip spectrum in the neighbourhood of the edge between 20-28 $\AA$ together with the model is shown in Fig. 5. Fitting in this range with a single power law for the continuum, we find an optical depth in the edge equivalent to a column density $N_{\mathrm{O}}=$ $(1.50 \pm 0.04) \times 10^{18} \mathrm{O}$-atom cm $\mathrm{cm}^{-2}$ and $(1.52 \pm 0.04) \times 10^{18} \mathrm{~cm}^{-2}$ for the non-dip and integrated dip spectra. However, the systematic error in the column density derived from a local fit to the continuum may exceed the statistical uncertainty. Thus, modelling the 20-38 A range, needing two powerlaws for the continuum, we obtain $N_{\mathrm{O}}=1.75_{-0.08}^{+0.07} \times 10^{18} \mathrm{O}$-atom $\mathrm{cm}^{-2}$ and $1.76_{-0.08}^{+0.07} \times 10^{18} \mathrm{~cm}^{-2}$ for the non-dip and dip spectra. Assuming an abundance of oxygen in the interstellar medium of $4.9 \times 10^{-4}$ (Wilms et al. 2000), this latter oxygen column corresponds to an equivalent hydrogen column $N_{\mathrm{H}}$ of $3.57_{-0.17}^{+0.14} \times 10^{21}$ atom $\mathrm{cm}^{-2}$ in non-dip, the value remaining unchanged in the integrated dip

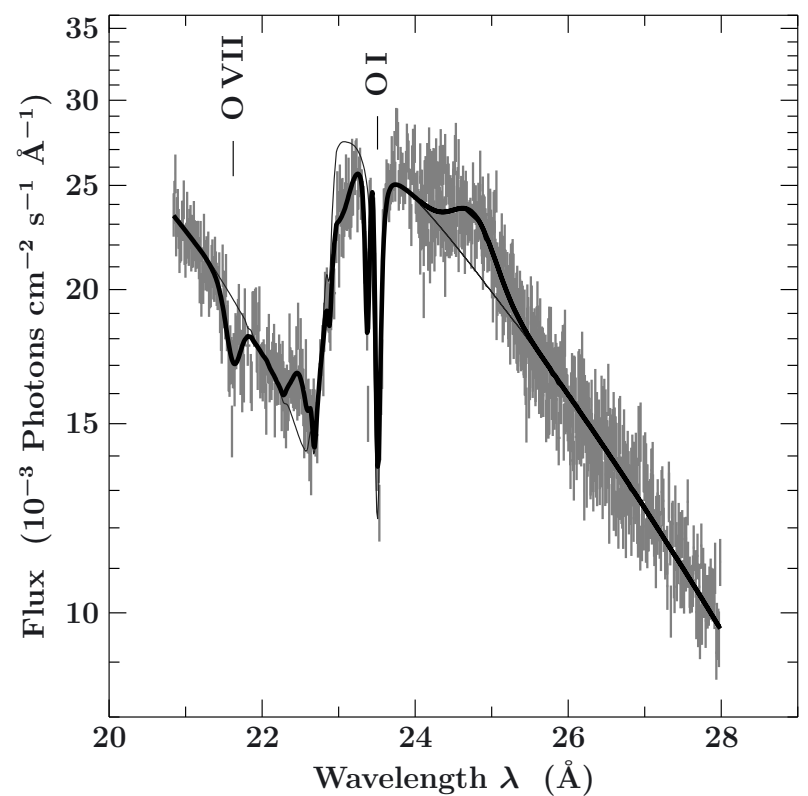

Fig. 5. First order RGS spectrum in the region of the oxygen edge, together with the best-fit model.

spectrum $\left(3.58_{-0.17}^{+0.14} \times 10^{21}\right.$ atom $\left.\mathrm{cm}^{-2}\right)$. Thus, this fitting supports the EPIC result that in dipping only uncovered emission is seen and $N_{\mathrm{H}}$ reflects interstellar absorption. The above values of $N_{\mathrm{H}}$ are in excellent agreement with that found in the EPIC-pn.

\subsection{Chandra grating results}

We now present results from the Chandra gratings for the present observation, and also compare these in Sect. 4.4.1 with all previous Chandra grating results for Cygnus X-2. We can use the HETGS data to investigate photoelectric absorption during persistent emission and during dips. We detect absorption edges for $\mathrm{Mg} \mathrm{K}, \mathrm{Ne} \mathrm{K}, \mathrm{Fe} \mathrm{L}$, and $\mathrm{O} \mathrm{K}$ at $9.48 \AA(1308 \mathrm{eV})$, $14.29 \AA(867 \mathrm{eV}), 17.51 \AA(708 \mathrm{eV})$, and $22.89 \AA(542 \mathrm{eV})$ (Juett et al. 2004, 2006). Since the Chandra data were taken in CC graded mode, we cannot model the spectral continuum as we did for the pn-spectra, but need to defer to a local continuum modelling. The lightcurve from the summed Chandra first order grating data is shown in the lower middle panel of Fig. 1. It excellently matches the pn-lightcurve from 4 to $71 \mathrm{ks}$. Note, that on top of heavy dipping activity, the overall persistent flux decreases by about 5\% over the entire exposure and instead of a simple cut by rate, we hand-selected and accumulated about $13 \mathrm{ks}$ of persistent events. Similarly, the dip minima vary considerably and again we selected and accumulated about $10 \mathrm{ks}$ of dip events from the bottoms of the dips. This selection results in a further reduction of the already reduced statistics with respect to the EPIC-pn, and we had to accumulate and add as many persistent and dipping intervals as possible throughout the lightcurve.

The left side of Fig. 6 shows broadband fits to the non-dip data (upper panel) and dip data (lower panel) using a multicomponent continuum model consisting of a power law and a blackbody component and the TBNEW high-resolution absorption function. Here we only use spectra from the MEG -1st order due to some hot pixel contamination in the $\mathrm{O} \mathrm{K}$ edge in the MEG +1 st order, which in CC-mode is difficult to remove. The fit in the persistent emission provides fair matches to the edges with a hydrogen equivalent of $(2.5 \pm 0.3) \times 10^{21} \mathrm{~cm}^{-2}$ for $\mathrm{O} \mathrm{K}$ and 
Cyg X-2, OBSID 10881, $70 \mathrm{ks}, \mathrm{MEG}-1$
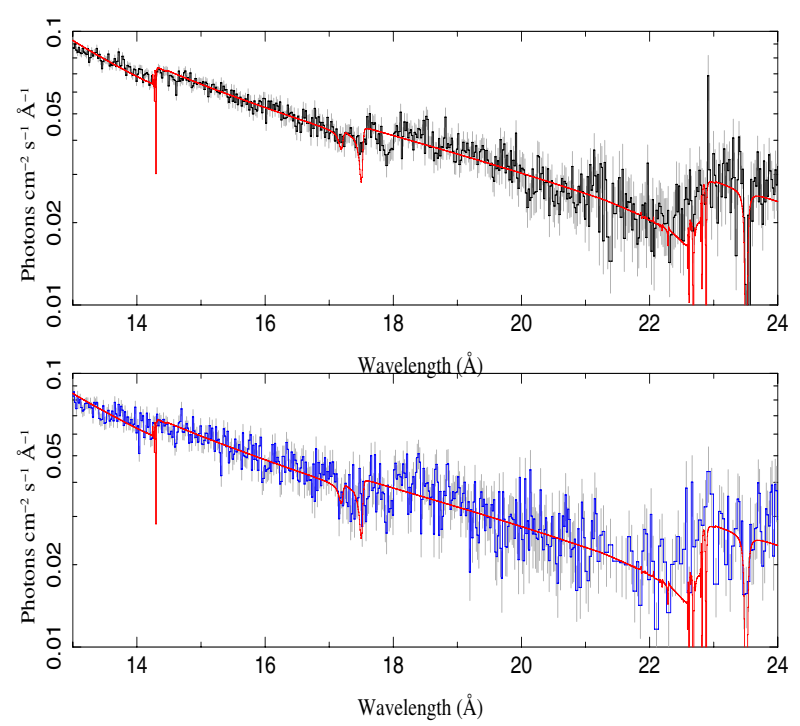

Cyg X-2, OBSID 10881, $70 \mathrm{ks}, \mathrm{MEG}+\mathrm{HEG}$
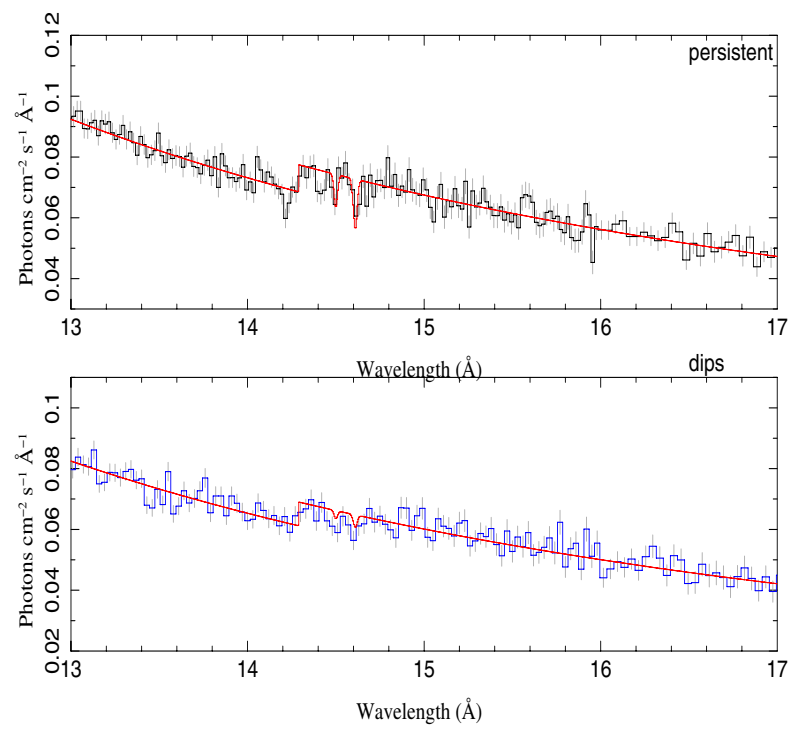

Fig. 6. Left: broadband fit using a multi-component continuum model and the TBNEw high-resolution absorption function to the MEG -1st order spectra of the persistent (top) and dip (bottom) emissions. The bandpass includes the Ne K edge at $14.29 \AA$ ( $867 \mathrm{eV}$ ), the Fe L II and III edges at $17.2 \AA(721 \mathrm{eV})$ and $17.5 \AA(708 \mathrm{eV})$, and the $\mathrm{O} \mathrm{K}$ edge at $22.89 \AA(542 \mathrm{eV})$. Right: the local fit to the Ne K edge using a simple power law and an edge function with an energy fixed at $14.29 \AA$.

Fe L, the one at Ne K appears higher at $(3.0 \pm 0.3) \times 10^{21} \mathrm{~cm}^{-2}$. The value from the oxygen edge is smaller than seen in the $X M M$ RGS and it is possible that this is due to residual crosscalibration uncertainties. The fits to the persistent and dip emission are practically identical as seen in the RGS, and the dip spectra do not seem to require adjusted optical depths, and we emphasize the surprizing nature of these results given the clear reduction of continuum intensity in dipping.

The optical depth at Ne K is much better determined because it provides the best statistics. Here we can use all the MEG data and the contributions from the HEG. We thus focus on local fits to the optical depth, and for this we simply apply an almost flat power law plus an edge function, which we fix at the expected rest value of the edge at $867 \mathrm{eV}(14.29 \AA)$. The results are shown in Fig. 6 (right panel). The scatter in the persistent and the dip spectrum is quite comparable and it is already visible that the edge depths are very similar. As described above, the equivalent hydrogen column density derived from the optical depth of the $\mathrm{Ne} \mathrm{K}$ edge appears slightly higher than for other edges. In order to investigate this we analysed the $\mathrm{Ne} \mathrm{K}$ edge region in several ways. In addition to the persistent and dip segments, we also determined the edge depth for the entire observation. This gave equivalent $N_{\mathrm{H}}$ values of $(3.40 \pm 0.96) \times 10^{21}$ atom $\mathrm{cm}^{-2}$ for the non-dip data, $(3.08 \pm 0.79) \times 10^{21}$ atom $\mathrm{cm}^{-2}$ for deep dipping and $(3.03 \pm 0.30) \times 10^{21}$ atom $\mathrm{cm}^{-2}$ when all data were added. The non-dip value is in good agreement with the $N_{\mathrm{H}}$ values from EPIC-pn and the RGS.

\subsubsection{Comparison with previous Chandra observations of Cyg X-2}

We also repeated the same procedure using previous HETGS data of Cyg X-2, i.e. obsid 1102 for 29 ks (Juett et al. 2004), obsid 1016 for $14.6 \mathrm{ks}$, and obsids 8170 and 8599 for $134 \mathrm{ks}$ (Schulz et al. 2009). The results are shown in Table 2 and Fig. 7 , and the results for the present observation are added to these. Our latest values agree with the previous values within uncertainties.

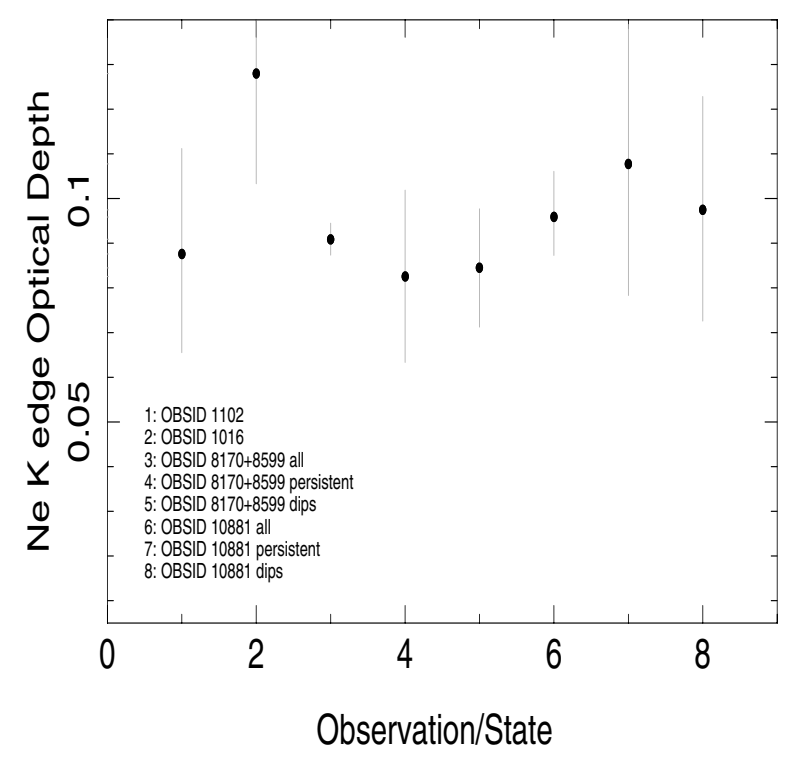

Fig. 7. Optical depth measurements of Ne K edges for all HETGS observations in the Chandra archive.

Juett et al. (2004) derived a Galactic column density for Cygnus X-2 from the oxygen edge of $N_{\mathrm{H}}=(2.3 \pm 0.5) \times$ $10^{21}$ atom $\mathrm{cm}^{-2}$, and Juett et al. (2006) obtained a column from the Ne edge $N_{\mathrm{Ne}}$ of $\left(2.3_{-0.3}^{+0.9}\right) \times 10^{17}$ atom $\mathrm{cm}^{-2}$ equivalent to a hydrogen column density of $\left(2.64_{-0.34}^{+1.0}\right) \times 10^{21}$ atom $\mathrm{cm}^{-2}$ (see also Yao et al. 2009). From our re-examination of the fitting of the $\mathrm{Ne}$ edge in these data (obsid 1102) we find $N_{\mathrm{H}}=$ $(2.77 \pm 0.72) \times 10^{21}$ atom $\mathrm{cm}^{-2}$ (Table 2 and Fig. 7$)$, in good agreement with the published value. It can be seen that the non-dip $N_{\mathrm{H}}$ value determined in the present work (Table 2) is higher but consistent with these values (as is the non-dip value of $(3.0 \pm 0.3) \times 10^{21}$ atom $\mathrm{cm}^{-2}$ determined from a reduced amount of data for $\mathrm{Ne} \mathrm{K}$ ). Moreover, the value obtained from the 
Table 2. Ne K optical depth and equivalent hydrogen column $N_{\mathrm{H}}=N_{\mathrm{Ne}} / 8.71 \times 10^{-5}$ from local fits during persistent emission and dipping.

\begin{tabular}{lrrrr}
\hline \hline State & Date (Obsid) & $\tau_{\mathrm{Ne}}$ & $\begin{array}{r}N_{\mathrm{Ne}} \\
\left(10^{17} \mathrm{~cm}^{-2}\right)\end{array}$ & $\begin{array}{r}N_{\mathrm{H}} \\
\left(10^{21} \mathrm{~cm}^{-2}\right)\end{array}$ \\
\hline All & 1999 Sep. 23 (1102) & $0.087 \pm 0.023$ & $2.41 \pm 0.63$ & $2.77 \pm 0.72$ \\
All & 2001 Aug. 12(1016) & $0.128 \pm 0.025$ & $3.52 \pm 0.69$ & $4.04 \pm 0.80$ \\
All & 2007 Aug. 23, 25 & $0.091 \pm 0.004$ & $2.50 \pm 0.10$ & $2.87 \pm 0.11$ \\
Non-Dip & $(8170+8599)$ & $0.083 \pm 0.019$ & $2.27 \pm 0.53$ & $2.61 \pm 0.61$ \\
Deep dip & & $0.084 \pm 0.013$ & $2.32 \pm 0.36$ & $2.67 \pm 0.42$ \\
All & present obs & $0.096 \pm 0.009$ & $2.64 \pm 0.26$ & $3.03 \pm 0.30$ \\
Non-Dip & 2009 May 12 & $0.108 \pm 0.030$ & $2.96 \pm 0.83$ & $3.40 \pm 0.96$ \\
Deep dip & $(10881)$ & $0.097 \pm 0.025$ & $2.68 \pm 0.69$ & $3.08 \pm 0.79$ \\
\hline
\end{tabular}

Notes. All means all integrated data, non-dip depicts flat and dip-free parts of the persistent emission, and deep dip the parts at the bottom of dips.

Fe L edge (above) of $(2.5 \pm 0.3) \times 10^{21}$ compares very well with $\left(2.64_{-0.34}^{+1.03}\right) \times 10^{21}$ atom $\mathrm{cm}^{-2}$ obtained by Juett et al. (2006). Thus this also suggests that no change has taken place. The difference between $\mathrm{Ne}$ and $\mathrm{Fe}$ values may indicate an underabundance of $\mathrm{Fe}$, i.e. a low $\mathrm{Fe} / \mathrm{Ne}$ ratio as suggested by Juett et al. (2006). If we were to assume that the larger value in the present work from the Ne edge of $(3.40 \pm 0.96) \times 10^{21}$ atom $\mathrm{cm}^{-2}$ and the value of Juett et al. (2004) from the oxygen edge are actually different, the implication could be that oxygen is depleted; however, Juett et al. (2004) found that the $\mathrm{O} / \mathrm{Ne}$ ratio in several sources was consistent with interstellar abundances; i.e. they found no evidence for departure.

However, although the sizes of the uncertainties in column density in general indicate that there is no significant evidence that $N_{\mathrm{H}}$ has changed, one point, that of obsid 1016, has a higher optical depth and a column density of $(4.04 \pm 0.80) \times$ $10^{21}$ atom $\mathrm{cm}^{-2}$, which is inconsistent with the point that follows it in Fig. 7. As the Galactic column density cannot change, this provides some indication that there may be a low level of intrinsic absorption. Evidence for intrinsic absorption was seen in Cygnus X-2 when in a much more active state by BałucińskaChurch et al. (2010), $N_{\mathrm{H}}$ increasing between the soft and hard apex. Evidence for a trend was clear, although the PCA instrument sensitive above $3 \mathrm{keV}$ is not well suited for accurate determinations of column density.

We also looked at the possibility of determining changes in line emissions during the persistent and dipping intervals. We detect all the lines described by Schulz et al. (2009) at line fluxes that are reduced to an approximately similar fraction as the continuum flux. For the selected persistent and dipping intervals the statistics are then already so low that we could not engage in a meaningful differential analysis. An overall analysis of the lines and a comparison with previous detections will be made in a future study.

\subsection{XMM OM results}

In Fig. 1 we compare the lightcurves of the Optical Monitor (lower panel) and the EPIC-pn (upper panel). Careful examination shows multiple occasions when X-ray dipping in the PN lightcurve is also seen in the OM lightcurve. For example, at $\sim 42 \mathrm{ks}$, there are definite flux decreases in both bands at 38.6, 40.4, 43.3 and $45.7 \mathrm{ks}$. Decreases are seen in both bands at 61.2 and $62.0 \mathrm{ks}$ and similar correspondences can be seen across the observation. The strong decreases in UV at $3 \mathrm{ks}$ and $30 \mathrm{ks}$ are matched by only weak decreases in the PN, suggesting that other factors affect the UV emission. At times later than $\sim 50 \mathrm{ks}$, the

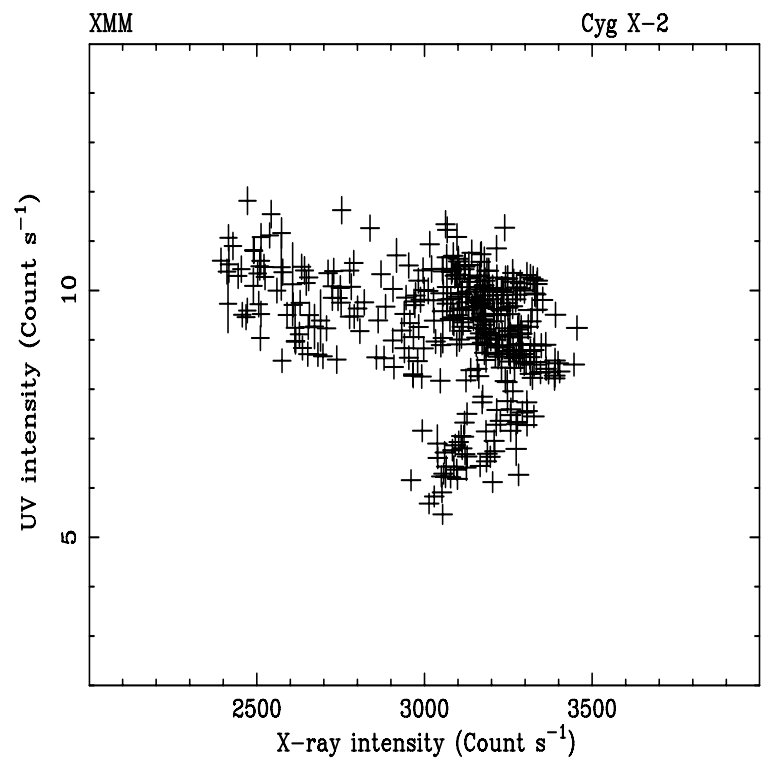

Fig. 8. Variation of Optical Monitor intensity in the UV band 2500-4000 $\AA$ with the X-ray intensity in the EPIC-pn $0.2-10.0 \mathrm{keV}$ band.

X-ray intensity drifts downwards while the OM lightcurve drifts upwards, and attempts to derive formally a correlation coefficient between $\mathrm{OM}$ and $\mathrm{X}$-ray data are affected by this. However, this is the first time that X-ray dipping in a LMXB has been seen in an optical or UV lightcurve. The reduction of UV intensity during X-ray dips shows that a substantial part of the emission originates in the accretion disk and it thus appears that the absorbing material removes both X-ray and UV emission.

In Fig. 8 we show the variation of OM intensity with the EPIC-pn $0.2-10 \mathrm{keV}$ intensity, although this is confused by the drifts mentioned above. The X-ray dipping from 3400 to 2500 count $\mathrm{s}^{-1}$ results in OM decreases from 10 to 9 count $\mathrm{s}^{-1}$ forming data with a slight slope in the figure. However, the drifts in both optical and X-ray intensities broaden this branch with increased OM intensity at lower X-ray intensities, masking the correlation. The other effect seen at $30 \mathrm{ks}$ of a deeper optical decrease for a smaller X-ray dip forms the second branch with a clear correlation. There may be an intrinsic variation in source intensity at this time so that a dependence of the optical signal on the X-ray intensity would indicate reprocessing of the X-ray emission. In any event, the small increase in the hardness ratios in Fig. 2 at this time shows that weak X-ray dipping takes place 
and the existence of two branches in Fig. 8 suggests that two different processes are occurring.

\subsection{Search for $Q P O$}

The EPIC-pn burst mode data were used to make a search for QPO. To avoid intoducing aliases, the data were rebinned to $3.90625 \mathrm{~ms}$, an exact multiple of the read-out time. Barycentric corrected data were given standard filtering (Sect. 2.1) and lightcurves were extracted using XMMSELECT and converted to power spectra using POWSPEC. This gave an accessible frequency range of $10^{-1}$ to $128 \mathrm{~Hz}$. The possible presence of $\mathrm{kHz}$ QPO could not be investigated because of the limits imposed by the binning required. The data were analysed in several ways. Firstly, the complete observation was searched for QPO, divided into 16-second segments. The observation was also divided into 4096-second sections of data in each of which an average power spectrum was extracted from shorter segments of data. Above $20 \mathrm{~Hz}$ a number of spurious peaks of instrumental origin exist derived from the read-out time and other binnings. No significant QPO detections were found in the power spectra, although there was a possible weak peak at $~ 5 \mathrm{~Hz}$.

\section{Discussion}

\subsection{Position on the Z-track}

During the observations the source did not move appreciably along the Z-track, as can be seen from the lack of intrinsic variation of the X-ray intensity in the EPIC-pn lightcurve (Fig. 1), confirmed by the lack of movement in the hardness-intensity diagram (Fig. 2). The only variability is due to the dipping and the results clearly show that the dips are due to partial absorption events and are not source changes. Moreover, the lightcurve exhibits a lack of flaring, which would be seen as intensity increases lasting a few thousand seconds so that the source is not on the flaring branch. Indeed, in many observations Cyg X-2 does not exhibit a traditional FB in the hardness-intensity diagram, although this is occasionally seen. In addition there can be intensity drops approximately at the soft apex, which form a distinct extra track in hardness-intensity. In the present observation, the neutron star blackbody temperature is low at $\sim 1.3 \mathrm{keV}$ and previous work has shown that such low values are found in the Cygnus X-2 like Z-sources at or near the soft apex, $k T_{\mathrm{BB}}$ increasing to $\sim 2 \mathrm{keV}$ at the hard apex and higher on the HB (e.g. Bałucińska-Church et al. 2010). Moreover, we measure a blackbody radius $R_{\mathrm{BB}}$ of $9.6 \pm 1.1 \mathrm{~km}$, indicating that the whole neutron star is emitting, again as found in previous work to occur at the soft apex. It thus appears that the source remains in a fixed position on the Z-track in the neighbourhood of the soft apex and that dipping is seen but not flaring. Previous studies suggested that dipping was a characteristic of the flaring branch (Sect. 1; Hasinger et al. 1990; Kuulkers et al. 1996), however, we now show that dipping and flaring are distinctly different phenomena. In terms of the model proposed for the Z-track sources based on the extended $\mathrm{ADC}$, the low value of $k T_{\mathrm{BB}}$ suggests a low and stable mass accretion rate $\dot{M}$; an increase would drive the source towards the hard apex and higher $k T_{\mathrm{BB}}$. It appears that $\dot{M}$ is indeed low, as the luminosity of the source is $\sim 30 \%$ less than in the previous observations of 2007 (Schulz et al. 2009). It is apparent that dipping is not a feature of the FB and furthermore, if flaring is due to unstable nuclear burning as proposed in the extended ADC model, and not due to $\dot{M}$ increase, there is no motivation for the suggestion that dipping is caused by inflation of the inner disk by radiation pressure at increased $\dot{M}$ (Kuulkers \& van der Klis 1995).

The distinction between dipping and flaring is important to a fundamental question relating to the Z-track sources. In a previous multi-wavelength campaign (Hasinger et al. 1990), Cygnus X-2 was observed in X-ray, ultraviolet, optical and radio. The ultraviolet results (Vrtilek et al. 1990) showed an increase of intensity on the FB and was a major argument for the widely accepted view that the mass accretion rate increases on the Z-track monotonically in the sense HB-NB-FB. However, the hardnessintensity diagram (Fig. 2 of Hasinger et al. 1990) viewed in the light of the present results clearly shows that the source variation was not flaring, but dipping. Thus the major argument for $\dot{M}$ increasing on the FB disappears, as does the contention that $\dot{M}$ increases from HB-NB-FB. In the Z-track source model based on an extended ADC (above) it is argued that $\dot{M}$ does not change in flaring but increases between the soft and hard apex, in the opposite sense to that in the standard view.

The lack of detection of radio emission is entirely consistent with this position on the Z-track: radio detection from relativistic jets is known to be concentrated at around the hard apex, and little emission is expected at the soft apex. In fact, it seems more plausible to us that any weak detection of radio on the lower normal branch may be a residual from the source having previously been at the hard apex. Spectral analysis of the Cyg-like sources as a function of Z-track position has previously shown that the $\mathrm{X}$-ray flux in the neighbourhood of the neutron star becomes several times the Eddington flux on the $\mathrm{HB}$. The strong correlation between this flux ratio $f / f_{\text {Edd }}$ and radio detection thus suggested that radiation pressure is responsible for jet launching via disruption of the inner disk (Church et al. 2006).

\subsection{The nature of intensity dips}

The lack of intrinsic spectral variation of the source during our observation is rather fortunate because it allows the study of the dipping events without interfering spectral continuum changes. Furthermore, we can take advantage of the broadband properties of the XMM EPIC-pn and the high-resolution Chandra and $X M M$ gratings in specific bandpasses. The EPIC-pn lightcurve shows dipping to remove only a fraction of the total luminosity $(\sim 25 \%)$ and we can immediately infer that the absorber covers the X-ray source only partially, which in turn must also be extended. The results of spectral fitting to the EPIC-pn data demonstrate for the first time that dipping in Cygnus X-2 is due to very strong partial absorption. The spectrum is well-fitted by blackbody emission plus Comptonized emission, which we associate with the neutron star and the extended ADC, respectively. In addition, broad line emission is seen at $\sim 1 \mathrm{keV}$ and at $\mathrm{Mg}$ XII, Si XIV, S XVI, and Fe XXV. The precise origin of the $1 \mathrm{keV}$ line is still unknown and part of ongoing analysis. The Comptonized emission dominates the X-ray flux, comprising $68 \%$ of the flux in the band $0.5-10 \mathrm{keV}$. The intermediate and deep dip spectra are well-fitted by a model in which the extended Comptonized emission of the ADC is progressively overlapped by the absorber, and the covering factor rising to 0.4 in deep dipping with an associated column density of $4.5 \times 10^{23}$ atom $\mathrm{cm}^{-2}$. In fact, fitting does not permit absorption of the blackbody emission and this is remarkable, given that in the dipping class of LMXB in which dips are caused by absorption in the outer disk bulge, the neutron star blackbody emission is always removed (e.g. Church et al. 1997). The dominant Comptonized emission in the present observation is removed gradually as dipping proceeds, i.e. it is partially covered with the covering factor increasing. 
This provides further strong support for the extended nature of the ADC in Cygnus X-2: part of the emitter being uncovered by absorber is not possible without an extended source. In the dipping LMXB such as XB 1916-053 (Church et al. 1997), the same process of progressive covering of an extended ADC also takes place, which is generally accepted as being due to absorption in the bulge in the outer accretion disk. However, the suggestion that the dipping LMXB sources can be explained by an ionized absorber at some location closer to the neutron star, removing point-like Comptonized emission (Boirin et al. 2005; Díaz Trigo et al. 2006) must be viewed with caution given the now extensive evidence for the extended ADC.

The Chandra gratings were used to provide high-quality spectra in the range 13-24 $\AA$ for both non-dip and deep dip data, revealing the Fe L II (17.2 $⿱$ ) and L III (17.5 $\AA$ ) edges, the O K edge $(22.89 \AA)$ and the Ne K edge at $14.29 \AA$. In the Chandra data the Ne edge has the best contrast with respect to the continuum and an effort was made to compare with results from previous observations. Most remarkable is the result that no difference in optical depth can be found between non-dip and deep dip spectra. The optical depth in the deep-dip spectra is $0.097 \pm$ 0.025 , which is practically identical with the non-dip value of $0.108 \pm 0.030$. The result is confirmed for the case of the $\mathrm{OK}$ edge in the RGS spectra. Given the large decrease in continuum intensity during the dips and the associated large value of partial $N_{\mathrm{H}}$ found from the EPIC-pn, a substantial increase in optical depth might have been expected. The fact that this is not found places severe constraints on a physical explanation.

We have derived the orbital phase range of the present observations using the ephemeris of Casares et al. (2010) with an orbital period of 9.84450 days to be $0.324 \pm 0.007-0.425 \pm 0.007$. This phase range is well outside the expected phase position of 0.75 where the disk bulge intercepts the line-of-sight between the X-ray emitting regions and the observer. We can thus rule out the bulge as the absorber, however, the actual phase of $\sim 0.35$ agrees with a position on the opposite side of the disk to the bulge where the accretion flow from the companion impacts. Interdipping is seen in dipping sources such as XB 1916053 with smaller dips between the main dips repeating at the orbital cycle (Church et al. 1997), caused possibly by ellipticity in the disk or by material overshooting the disk creating a secondary bulge. In the present case, we may be seeing interdipping, or possibly we may see dipping caused by clouds of material traversing the system above the accretion disk. The inclination of the Cygnus X-2 system is known to be $62 .^{\circ}$ (Cowley et al. 1979), a little outside the range of inclination angles of $65-85^{\circ}$ normally associated with the dipping LMXB. At this inclination, we would expect an extended ADC to be partly overlapped by absorber either at the main bulge or secondary bulge, but that the vertical height of absorber would just miss overlapping the neutron star, thus explaining the lack of absorption of the blackbody emission. Clouds of absorber traversing above the disk could also cover part of the ADC without covering the neutron star. Dipping appears entirely as absorption of the Comptonized emission. The fraction of this emission covered by absorber is strongly absorbed with a high column density $N_{\mathrm{H}} \sim 4.5 \times 10^{23}$ atom $\mathrm{cm}^{-2}$ removing all flux below $4 \mathrm{keV}$. Thus, in the grating spectra a reduction of continuum intensity is seen in dipping, but we only see the $60 \%$ of the emission that is not covered at all, and this explains why there is no increase in the measured optical depth in the edges.

We can estimate the size of absorber from dip ingress times. If dipping is caused by absorption in a secondary bulge in the outer accretion disk, the dip ingress time $\Delta t$ is the time needed for the absorber of diameter $D$ and velocity $v$ to overlap the extended emitter, so that $\Delta t=D / v$. The velocity at which the bulge moves is $2 \pi R / P$, where $P$ is the orbital period, and for the measured $\Delta t$ of $\sim 750 \mathrm{~s}$, the absorber size $D$ is $35000 \mathrm{~km}$. Schulz et al. (2009) give a radial size of the ADC in Cyg X-2 of $110000 \mathrm{~km}$, so that the absorber would convincingly give a covering factor of about $40 \%$, as observed.

If the absorbers were clouds traversing above the disk, these may be blobs of material originating where the stream from the companion strikes the outer disk forming the bulge. Overflowing of the disk has been suggested by several authors. Armitage \& Livio (1996) investigated theoretically the disturbance to the accretion flow at the point of impact resulting in material being moved significantly out of the orbital plane although this was thought to return to the orbital plane and not cross the disk to the opposite side. Assuming a typical infall velocity that will be much higher than the bulge velocity (above) would lead to a very large absorber size $D=v \Delta t$, several times larger than for the bulge (above), so giving $100 \%$ dips, much deeper than we observe.

\subsection{Is there a dipping state?}

Last, but not least, we would like to address the issue of why we observe these dipping events in Cygnus X-2 when the source is near the soft apex. In the previous Chandra observation the dipping occurred at the soft apex (Schulz et al. 2009), in the present one the derived blackbody temperature and radius imply a similar location on the Z-track, albeit at an overall lower flux. The persistent X-ray flux is dropping at a constant rate indicating the source may still be moving to a lower flux. At the soft apex, the spectra appear particularly soft and the X-ray intensity is low.

The reason why the overall intensity in our observation is much lower is not clear although it may well be due to reduced mass accretion rate. We can, however, exclude enhanced absorption. Figure 7 compares the optical depths of the Ne K edge for previous high-resolution observations and as discussed previously, there is no significant increase in the present observation. The values obtained in non-dip from the three instruments: $(3.6 \pm 0.1) \times 10^{21}($ EPIC-pn $),\left(3.57_{-0.17}^{+0.14}\right) \times 10^{21}(\mathrm{RGS})$ and $(3.4 \pm 0.96) \times 10^{21}($ Chandra $)$ atom $\mathrm{cm}^{-2}$ agree well. If we were to assume that the difference between these values and the Juett et al. (2004) value of $(2.3 \pm 0.5) \times 10^{21}$ atom cm $^{-2}$ was real, we find from our EPIC-pn spectral fit to non-dip emission that increasing $N_{\mathrm{H}}$ from the Juett value to the EPIC value causes the $1-10 \mathrm{keV}$ luminosity to decrease by only $5 \%$ so it is ruled out that the decrease in luminosity since the previous Chandra observation is due to enhanced absorption.

The fact that we often observe dipping at the soft apex might simply indicate that we observe a switch from a mostly ionized plasma on the normal branch and horizontal branch to the regaining of ionization fractions at lower luminosities. At the soft apex the plasma begins to recombine even to the point of neutral material. For recombination coefficients of $\sim 10^{-11} \mathrm{~cm}^{3} \mathrm{~s}^{-1}$ and plasma densities above $10^{12} \mathrm{~cm}^{-3}$ this should happen in a fraction of a second. However, detailed modelling would be required to fully understand the ionization balance in such a scenario. In summary, it remains unclear why dipping appears concentrated at this part of the Z-track.

Acknowledgements. We thank the participants in the e-EVN radio observations at Cambridge, Jodrell Bank, Knockin, Effelsberg, Medicina, Torun, Onsala, 
Sheshan and Yebes. This work was supported in part by the Polish Ministry of Science and Higher Education grant 3946/B/H03/2008/34.

\section{References}

Armitage, P. J., \& Livio, M. 1996, ApJ, 470, 1024

Bałucińska-Church, M., Gibiec, A., Jackson, N. K., \& Church, M. J. 2010, A\&A, 512, A9

Bildsten, L. 1998, in Proc NATO ASIC 515, The Many Faces of Neutron Stars, ed. R. Buccheri, J. van Paradijs, \& M. A. Alpar (Dordrecht-Kluwer), 419

Boirin, L., Méndez, M., Díaz Trigo, M., Parmar, A. N., \& Kaastra, J. S. 2005, A\&A, 436, 195

Bonnet-Bidaud, J. M., \& van der Klis, M. 1982, A\&A, 116, 232

Brandt, W. N., \& Schulz, N. S. 2000, ApJ, 544, L123

Casares, J., Charles, P. A., \& Kuulkers, E. 1998, ApJ, 493, L39

Casares, J., González-Hernández, J. I., Israelian, G., \& Rebolo, R. 2010, MNRAS, 401, 2517

Church, M. J., \& Bałucińska-Church, M. 1997, ApJ, 491, 388

Church, M. J., \& Bałucińska-Church, M. 2004, MNRAS, 348, 955

Church M. J., Halai G. S., \& Bałucińska-Church, M. 2006, A\&A, 460, 233

Cowley, A. P., Crampton, D., \& Hutchings, J. B. 1979, ApJ, 231, 539

den Herder, J. W., Brinkman, A. C., Kahn, S. M., et al. 2001, A\&A, 365, L7

di Salvo, T., Farinelli, R., Burderi, L., et al., 2002, A\&A, 386, 535

Done, C., Życki, P., \& Smith, D. A. 2002, MNRAS, 331, 453

Díaz Trigo, M., Parmar, A. N., Boirin, L., Méndez, M., \& Kaastra, J. S. 2006, A\&A, 445, 179

Elebert, P., Callanan, P. J., Torres, M. A. P., \& Garcia, M. R. 2009, MNRAS, 395, 2029

Fender, R., Wu, K., Johnston, H., et al. 2004, Nature, 427, 222

Fomalont, E. B., Geldzahler, B. J., \& Bradshaw, C. F. 2001, ApJ, 558, 283

Hasinger, G., \& van der Klis, M. 1989, A\&A, 225, 79

Hasinger, G., van der Klis, M., Ebisawa, K., Dotani, T., \& Mitsuda, K. 1990 A\&A, 235, 131
Heinz, S., Schulz, N. S., Brandt, W. N., \& Galloway, D. K. 2007, ApJ, 663, L93 Hjellming, R. M., Han, X. H., Córdova, F. A., \& Hasinger, G. 1990, A\&A, 235, 147

Juett, A. M., Schulz, N. S., \& Chakrabarty, D. 2004, ApJ, 612, 308

Juett, A. M., Schulz, N. S., Chakrabarty, D., \& Gorczyca, T. W. 2006, ApJ, 648 1066

Kirsch, M. G. F., Schönherr, G., Kendziorra, E., et al. 2006, A\&A, 453, 173

Kuulkers, E., \& van der Klis, M. 1995, A\&A, 303, 801

Kuulkers, E., van der Klis, M., \& Vaughan, B. A. 1996, A\&A, 311, 197

Kuulkers, E., Parmar, A. N., Owens, A., Oosterbroek, T., \& Lammers, U. 1997, A\&A, 323, L29

Lin, D., Remillard, R. A., \& Homan, J. 2009, ApJ, 696, 1257

Mason, K. O., Breeveld, A., Much, R., et al. 2001, A\&A, 365, L36

Orosz, J. A., \& Kuulkers, E. 1999, MNRAS, 305, 1320

Rushton, A., Bach, U., Spencer, R. E., et al. 2009, Astronomer's Telegram, 2052

Schulz, N. S., Hasinger, G., \& Trümper, J. 1989, A\&A, 225, 48

Schulz, N. S., Huenemoerder, D. P., Ji, L., et al. 2009, ApJ, 692, L80

Shaposhnikov, N., Titarchuk, L, \& Laurent, P. 2009, ApJ, 699, 1223

Shapiro, S. L., Lightman, A. P., \& Eardley, D. M. 1976, ApJ, 204, 187

Shirey, R. E., Bradt, H. V., Levine, A. M., \& Morgan, E. H. 1998, ApJ, 506, 374

Smale, A. P. 1998, ApJ, 498, L141

Smale, A. P., Done, C., Mushotzky, R. F., et al. 1993, ApJ, 410, 796

Smale, A. P., Angelini, L., White, N. E., Mitsuda, K., \& Dotani, T. 1994, BAAS, 26,1484

Torrejón, J. M., Schulz, N. S., Nowak, M. A., \& Kallman, T. R. 2010, APJ, 715, 947

Turner, M. J. L., Abbey, A., Arnaud, M., et al. 2001, A\&A, 365, L27

Verner, D. A., Ferland, G. J., Korista, K. T., \& Yakovlev, D. G. 1996, ApJ, 465 487

Vrtilek, S. D., Swank, J. H., Kelley, R. L., \& Kahn, S. M. 1988, ApJ, 329, 289

Vrtilek, S. D., Raymond, J. C., Garcia, M. R., et al. 1990, A\&A, 235, 162

White, N. E., \& Swank, J. H. 1982, ApJ, 253, L61

Wilms, J., Allen, A., \& McCray, R. 2000, ApJ, 542, 914

Yao, Y., Schulz, N. S., Gu, M. F., Nowak, M. A., \& Canizares, C. R. 2009, ApJ, 696,1418 